\title{
DLL4 $^{+}$Dendritic Cells: Key Regulators of Notch Signaling in Effector T Cell Responses
}

\author{
Lijun Meng, ${ }^{1}$ Shaoyan $\mathrm{Hu},{ }^{2}$ Jian Wang, ${ }^{2}$ Shan $\mathrm{He}^{1}$ and Yi Zhang ${ }^{1,3,4}$
}

${ }^{1}$ Fels Institute for Cancer Research and Molecular Biology, Temple University, USA; ${ }^{2}$ Department of Hematology, Children's Hospital, Soochow University, Suzhou, China; ${ }^{3}$ Department of Microbiology and Immunology, Temple University, USA.

\section{Correspondence:}

${ }^{4}$ Yi Zhang, MD, PhD,

Fels Institute for Cancer Research and Molecular Biology

Department of Microbiology and Immunology

Temple University School of Medicine

Philadelphia, PA 19140, USA

Phone: 215-707-8901

Email: yi.zhang@temple.edu 


\begin{abstract}
:
Dendritic cells (DCs) are critical regulators of adaptive immune responses. DCs can elicit primary $\mathrm{T}$ cell responses at low $\mathrm{DC}: \mathrm{T}$ cell ratios through their expression of high levels of antigen-presenting molecules and costimulatory molecules. DCs are important for induction of functionally diverse $\mathrm{T}$ cell subsets such as $\mathrm{CD}^{+} \mathrm{T}$ helper (Th) 1 and Th17 cells and effector $\mathrm{CD}^{+} \mathrm{T}$ cells able to reside in epithelial tissues. Recent studies begin illuminating the underlying mechanism by which DCs regulate specialized T cell subsets. DCs are composed of subsets that differ in their phenotype, localization and function. DCs expressing high levels of DLL4 (DLL4 ${ }^{+}$ DCs), which is a member of Notch ligand family, are newly discovered cells that have greater ability than DLL4- DCs to promote the generation of Th1 and Th17 CD4 ${ }^{+}$T cells. DLL4 derived from DLL4 ${ }^{+}$DCs is also important for promoting the differentiation and expansion of effector $\mathrm{CD}^{+} \mathrm{T}$ cells. Experimental studies have demonstrated that selective deletion of DLL4 in DCs causes impaired antitumor immunity. In contrast, blocking DLL4 leads to dramatic reduction of inflammatory $\mathrm{T}$ cell responses and their-mediated tissue damage. We will discuss emerging functional specialization within the DLL4 ${ }^{+}$DC compartment, DLL4 ${ }^{+}$DC biology and the impact of pharmacological modulation of DLL4 to control inflammatory disorders.
\end{abstract}




\author{
Abbreviation: \\ APC: antigen presenting cell \\ CTL: cytotoxic T lymphocyte \\ DC: dendritic cell \\ cDC: conventional dendritic cell \\ pDC: plasmacytoid dendritic cell \\ CMP: common myeloid progenitor \\ HPC: hematopoietic progenitor cell \\ MDP: macrophage and DC progenitor \\ HSCT: hematopoietic stem cell transplantation \\ DLL: delta-like ligand \\ GVHD: graft-versus-host disease \\ NICD: Notch intracellular domain \\ moDC: monocyte-derived dendritic cell \\ Th: T helper \\ TLR: toll-like receptor \\ TCR: T cell receptor
}

Key words:

DLL4; DLL4 ${ }^{+}$DCs; Notch signaling; tumor immunity; alloimmunity and autoimmunity 


\section{CONTENTS}

\section{A. INTRODUCTION}

B. INDUCTION OF DLL4 ${ }^{+}$DCS AND LINEAGE-SPECIFIC EFFECTOR T CELLS B.1. DC expression of DLL4 under inflammatory conditions.

B.2. Induction of specialized T cell subsets by DLL4 ${ }^{+}$DCs.

C. DLL4 ${ }^{+}$DC ACTIVATION OF NOTCH SIGNALING IN T CELLS.

C.1. Notch signaling in effector $\mathrm{T}$ cell subsets.

C.2. High binding affinity between DLL4 and Notch1/4.

D. MECHANISMS THAT REGULATE DLL4 ${ }^{+}$DC DIFFERENTIATION

D.1. The role of Toll-like receptor (TLR) signaling in DC expression of DLL4

D.2. Cytokine-mediated fate determination of immature DLL4 ${ }^{+}$DCs.

E. REGULATION OF BY DLL4 IN DCS BY NFאB and STAT3

F. DLL4 AND DLL4 ${ }^{+}$DCS IN T CELL IMMUNITY

F.1. Autoimmunity

F.2. Tumor immunity

F.3. Alloimmunity

\section{G. FUTURE PERSPECTIVES}




\section{A. INTRODUCTION}

Dendritic cells (DCs) are essential for eliciting primary $\mathrm{T}$ cell responses. ${ }^{1-3}$ DCs express high levels of antigen presenting molecules, which present antigen peptides to prime naïve $\mathrm{T}$ cells by triggering $\mathrm{T}$ cell receptor (TCR) signaling, and costimulatory molecules, which are able to amplify TCR signaling to promote proliferation and survival of activated T cells. ${ }^{1-3}$ DCs also produce cytokines to direct effector differentiation, ${ }^{4-8}$ however, DCs can polarize the generation of distinct lineages of effector cells independent of cytokines. ${ }^{9,10}$ We now appreciate that Notch ligands expressed on the surface of DCs are crucial for promoting effector differentiation. ${ }^{11-14}$ In mammalians, there are five Notch ligands (i.e., Delta-like 1 (DLL1), DLL3, DLL4, Jagged1 and Jagged2) ${ }^{15-17}$ Notch ligands interact with Notch receptors (Notch 1, 2, 3, and 4), ${ }^{15,17,18}$ triggering the release of intracellular Notch and the subsequent transcription of Notch target genes. ${ }^{15,17,18}$ Among these Notch ligands, DLL4 shows greater capacity than others to promote the generation of $\mathrm{T}$ helper (Th)1 and Th17 $\mathrm{CD}^{+} \mathrm{T}$ cells, which are characterized by producing high levels of IFN- $\gamma$ and IL-17, respectively. ${ }^{14,19-21}$ This leads to our discovery of DLL4-positive (DLL4 ${ }^{+}$) DCs that have greater capacity than DLL4-negative (DLL4) DCs to induce Th1 and Th17 cells. ${ }^{14,19,20}$ A recent study reports that DC-derived DLL4 was able to regulate $\mathrm{CD} 4^{+} \mathrm{T}$ cell metabolism and proliferation through potentiating $\mathrm{T}$ cell receptor $(\mathrm{TCR}) / \mathrm{CD} 28$ signaling. ${ }^{22}$ Thus, DLL4 activation of Notch may represent a 'fourth' signal that is crucial for instructing effector development. ${ }^{11,12,14,19,20,23,24}$ This review will focus on discussing our current understanding of biology of DLL4 ${ }^{+}$DCs and their-derived DLL4 in the generation of specialized effector T cells and their-mediated inflammatory disorders.

\section{B. INDUCTION OF DLL4 ${ }^{+}$DCS AND LINEAGE-SPECIFIC EFFECTOR T CELLS}


Early studies revealed that DLL4 was highly and selectively expressed within vascular endothelium and crucial for the control of endothelial cell development. ${ }^{25}$ In 2004, studies by Amsen and colleagues suggested that DLL4 was induced in lipopolysaccharides (LPS)stimulated bone marrow (BM) cells that contained antigen-presenting cells (APCs). Ectopic expression of DLL4 in IEk-expressing L cell lines enhanced the production IFN- $\gamma$ by naïve CD4 ${ }^{+}$ T cells of AND TCR transgenic T cells. ${ }^{12}$ In 2007, Skokos and colleagues reported that CD8DCs induced IL-12-independent Th1 differentiation through DLL4 activation of Notch. ${ }^{10}$ Subsequent studies showed that DLL4 regulated pathogenesis of inflammatory diseases in experimental mice, including respiratory viral (RSV) infection, ${ }^{26,27}$ experimental allergic conjunctivitis, $^{28}$ experimental autoimmune encephalomyelitis ${ }^{29}$ and mycobacteria-elicited pulmonary granulomatous. ${ }^{30}$ These studies open the perspective to explore the importance of DLL4 and DLL4 ${ }^{+}$DCs in inflammatory T cell responses. However, under steady state conditions, DLL4 was barely detected on the surface of DCs and only a small numbers of DLL4 ${ }^{+}$DCs could be recovered from normal mice, ${ }^{10,26-28,30,31}$ which limited our capacity to investigate the biological properties of these DLL4 ${ }^{+}$DCs. By 2013, we discovered that DLL4 was dramatically upregulated on the surface of approximately $40 \%$ of host DCs from the spleen of mice undergoing preparative conditioning for allogeneic hematopoietic stem cell transplantation (HSCT). This finding allowed us to characterize these DLL4 ${ }^{+}$DCs. ${ }^{14}$ Most recently, we have established a novel culture system that can produce large amount of DLL4 ${ }^{+}$DCs from murine BM, providing a unique opportunity to investigate their biology and clinical implications. ${ }^{19}$ 
B.1. DC expression of DLL4 under inflammatory conditions. DCs are heterogeneous cell populations. ${ }^{32}$ Based on their surface phenotype, anatomical location and function, murine DCs at the steady state condition are broadly categorized into conventional DCs (cDCs, CD11 $\mathrm{c}^{+}$PDCA-1-B220-) and plasmacytoid DCs (pDCs, CD11 $\mathrm{c}^{+}$PDCA- $\left.1^{+} \mathrm{B} 220^{+}\right){ }^{32,33}$ Under inflammatory conditions, immature DCs profoundly change their phenotype, acquire enhanced antigen-presenting capacity and alter migration capability. ${ }^{33-37}$ To define how inflammatory DCs induce specialized effector T cells, we used murine models of allogeneic HSCT that cause graftversus-host disease (GVHD). In the GVHD model, recipient mice were pre-conditioned using lethal irradiation, followed by transfer of $\mathrm{BM}$ with or without $\mathrm{T}$ cells from allogeneic donors. Lethal irradiation would cause tissue injury and gastrointestinal (GI) dysfunction, leading to the release of danger signals and entry of LPS. Within three days after transplantation, DCs were all of host origin and expressed high levels of MHC class II molecule Ia and costimulatory molecules CD80 and CD86. ${ }^{14}$ Thus, DCs generated in hosts undergoing preparative conditioning resemble the phenotype of inflammatory DCs. . $3,34,36,37^{-3}$

The expression of DLL4 is induced in immature DCs upon inflammatory stimulation. Data from our studies and others indicate that under steady state condition in mice, only a small fraction of pDCs expressed low levels of DLL4, whereas cDCs did not produce DLL4. ${ }^{14,38}$ In contrast, DLL4 was dramatically upregulated on the surface of approximately $40 \%$ of murine whole DC population from the spleen of allogeneic HSCT recipients. Notably, while approximately $10 \%$ cDCs derived from mice undergoing HSCT also upregulated the expression of DLL4, as many as $80 \%$ of murine DLL4 ${ }^{+}$DCs derived from mice undergoing HSCT were PDCA- $1^{+} \mathrm{B} 220^{+}$cells, resembling to $\mathrm{pDCs}$ under steady state conditions. However, these DLL4 ${ }^{+}$pDC-like cells 
expressed CD11b, which is normally not seen in steady state pDCs. ${ }^{32}$ Upon inflammatory stimulation, immature DCs may alter their phenotype and functionality. ${ }^{33-35,39}$ Indeed, our subsequent studies showed that while immature pDCs derived from BM cells cultured in the presence of Flt3 ligand (referred to as FL) did not express high level of DLL4, they rapidly upregulated DLL4 transcript and protein upon activation of TLR signaling, accompanied with their increase of CD11b and other costimulatory molecules (e.g., CD40, CD80 and CD86). ${ }^{19}$ These observations suggest that DLL4 ${ }^{+}$pDC-like cells may be derived from immature $\mathrm{pDCs}$ upon inflammatory stimulation. Thus, both murine DLL4 $4^{+} \mathrm{pDCs}$ and $\mathrm{DLL} 4^{+} \mathrm{cDCs}$ occurred in mice under the allogeneic HSCT condition, with the former being a major subset. Notably, these DLL4 ${ }^{+}$cDCs did not express CD8. ${ }^{14,19}$

Our understanding of human DCs derived predominantly from studies of cells isolated from peripheral blood. ${ }^{40}$ Under steady state conditions, human peripheral blood (PB) DCs lack lineage (Lin) markers (i.e., CD3, CD15, CD19, CD14, CD20 and CD56) and constitutively express HLA-DR $\left(\mathrm{DR}^{+}\right)$, and can be broadly categorized into two major subsets: cDCs and pDCs. ${ }^{6}$ Human cDCs are characterized as Lin-HLA-DR ${ }^{+} \mathrm{CD} 11 \mathrm{c}^{+}$cells, whereas pDCs are Lin-HLA$\mathrm{DR}^{+} \mathrm{CD} 11 \mathrm{c}^{-} \mathrm{CD} 123^{+}$cells. $^{6,41}$ In $\mathrm{PB}$, cDCs can be further classified into at least two subsets: CD $1 \mathrm{c}^{+}$DCs and $\mathrm{CD} 141^{+}$DCs. ${ }^{42}$ The former comprises the predominant cDC subsets, whereas the latter is a relatively small population. We found that only a small fraction of CD1c $\mathrm{c}^{+} \mathrm{DCs}$ $(2.2 \%)$ and $\mathrm{pDCs}(0.8 \% \pm 0.2 \%)$ from PB of healthy donors expressed low levels of DLL4 on their surface. ${ }^{20}$ In contrast, allogeneic HSCT recipients had a 16 -fold more DLL4 ${ }^{+} \mathrm{CD} 1 \mathrm{c}^{+}$DCs (32.4\%) than healthy donors. Human pDCs (3.2\%) from HSCT patients also upregulated DLL4, but to a much less extent than $\mathrm{CD} 1 \mathrm{c}^{+} \mathrm{DCs} .^{20}$ These studies clearly identify the existence of 
human counterpart of mouse DLL4 ${ }^{+}$DCs. Furthermore, the expression of DLL4 represents a unique marker of activating DCs under inflammatory conditions.

B.2. Induction of specialized T cell subsets by $\operatorname{DLL4}^{+}$DCs. Induction of specialized $\mathrm{T}$ cell subsets is important for both protective and inflammatory immune responses. DLL4 derived from $\mathrm{CD} 1 \mathrm{c}^{+} \mathrm{DCs}$ is critical for the induction of Th1 and $\mathrm{Th} 17 \mathrm{CD}^{+} \mathrm{T}$ cells. In mixed lymphocyte reaction (MLR) cultures, activated DLL4 ${ }^{+}$DCs were better able to promote Th1 and Th17 differentiation than unstimulated PB DCs. Blocking DLL4 using a neutralizing antibody (Ab) decreased Notch signaling in $\mathrm{T}$ cells stimulated with $\mathrm{DLL}^{+} \mathrm{DCs}$, and reduced the generation of Th1 and Th17 CD4 ${ }^{+} \mathrm{T}$ cells. ${ }^{20}$ Like their human counterparts, murine DLL4 ${ }^{+}$DCs had greater ability than DLL4- DCs to promote the development of effector $\mathrm{CD}^{+} \mathrm{T}$ cells producing IFN- $\gamma$ and IL-17. ${ }^{14}$ Furthermore, in vivo administration of neutralizing anti-DLL4 Ab led to decreased generation of inflammatory Th1 and Th17 cells, and the reduction of GVHD in mice receiving allogeneic HSCT. Notably, blocking DLL4 had no significantly impact on regulatory T cells (Treg). ${ }^{14,19,24}$ Thus, DLL4 ${ }^{+}$DCs and their derived DLL4 play critical roles in regulating functionally specialized $\mathrm{CD} 4^{+} \mathrm{T}$ cell subsets.

Recent studies suggested that both human $\mathrm{CD} 1 \mathrm{c}^{+} \mathrm{DCs}$ and $\mathrm{CD} 141^{+} \mathrm{DCs}$ acquired influenza antigens in vivo and expanded specific cytotoxic $\mathrm{CD} 8^{+} \mathrm{T}$ cells in vitro using humanized mice infected by live-attenuated influenza virus (LAIV). ${ }^{43}$ Interestingly, lung-tissue-resident CD1c ${ }^{+}$ DCs but not $\mathrm{CD} 141^{+} \mathrm{DCs}$, were able to drive $\mathrm{CD} 103$ expression on $\mathrm{CD} 8^{+} \mathrm{T}$ cells and promoted $\mathrm{CD} 8^{+} \mathrm{T}$ cell accumulation in the lung epithelia in vitro and in vivo. ${ }^{43}$ Given the importance of DLL4 on human CD1c ${ }^{+}$DC-mediated IFN- $\gamma$, it will be intriguing to determine whether DLL4 
derived from $\mathrm{CD} 1 \mathrm{c}^{+}$DCs may play important roles in the generation of specialized effector $\mathrm{CD} 8^{+}$ $\mathrm{T}$ cells, such as the capacity to produce different profile of inflammatory cytokines and chemokines and the ability to express chemokine receptors and adhesion molecules. Data from our studies and others suggest that tissue resident DCs play important roles in regulating $\mathrm{T}$ cell function in the local tissues. ${ }^{43-48}$ In mice undergoing allogeneic HSCT, DLL4 ${ }^{+}$DCs were identified in the intestine where the occurrence of GVHD is associated with increased risk of mortality in recipients. ${ }^{14}$ Other studies suggested that tissue resident DCs expressing CD103 were important for expanding tumor-reactive $\mathrm{T}$ cells to sufficient numbers for controlling tumor growth. Better understanding of how tissue resident DLL4 ${ }^{+}$DCs influence T cell immunity in the local tissue may lead to new strategies of modulating alloimmunity and tumor immunity.

\section{DLL4 ${ }^{+}$DC ACTIVATION OF NOTCH SIGNALING IN T CELLS.}

C.1. Notch signaling in effector $\mathbf{T}$ cell subsets. Notch is a conserved signaling pathway that plays important roles in multiple cell processes, including cell growth, proliferation, differentiation and fate determination ${ }^{15-17}$. Notch receptor is composed of an extracellular ligandbinding domain, a single-pass transmembrane domain and an intracellular domain (Figure.1). Binding of Notch ligand to Notch receptors leads to its cleavage by $\gamma$-secretase complex, and release of active intracellular domain of Notch (NICD). NICD is translocated from cytoplasm into nucleus, where it binds to the DNA-binding protein complex CSL/RBP-J, recruits transcriptional co-activators including MAML and p300, and subsequently activates gene transcription. In peripheral immune responses, Notch signaling activates major transcription factors including T-bet, GATA3 and Rorc to instruct the differentiation of activated CD4 T cells 
into Th1, Th2 and Th17 cells, respectively. Recent studies suggested that DLL4 promoted CD4 ${ }^{+}$ T cells to secrete IL-17 via a mechanism of upregulating Rorc and I117 transcription. ${ }^{49-51}$

DLL4 $^{+}$DCs promote Th1 and Th17 polarization at least partially dependent on canonical Notch signaling. Data from our recent studies indicate that DLL4 ${ }^{+}$DCs promote effector differentiation via a Notch-dependent mechanism. Dominant-negative (DN)-MAML is a specific pan-Notch inhibitor. $^{52}$ Upon allogeneic DLL4 ${ }^{+} \mathrm{DC}$ activation, DN-MAML-expressing $\mathrm{CD}^{+} \mathrm{T}$ cells produced five-times fewer Th1 cells in cultures compared to wild-type (WT) $\mathrm{CD} 4^{+} \mathrm{T}$ cells. ${ }^{53}$ Addition of anti- DLL4 Ab did not further decrease Th1 cells in cultures of DN-MAML CD4 ${ }^{+} \mathrm{T}$ cells. Thus, Notch signaling is essential for DLL4 ${ }^{+}$DC-mediated Th1 differentiation. Anti-DLL4 $\mathrm{Ab}$ treatment reduced activation of Notch 1 signaling and downstream targets of Notch signaling, including Hes1 and Dtx1, in CD4 ${ }^{+} \mathrm{T}$ cells cocultured with DLL4 ${ }^{+}$DCs. This effect of anti-DLL4 $\mathrm{Ab}$ was associated with significantly decreased expression of Tbx21 and Rorc transcripts. ${ }^{14,19}$ Interestingly, an elegant study by Bailis and colleagues showed that Notch signaling was able to simultaneously orchestrate multiple helper $\mathrm{T}$ cell programs via a mechanism independently of cytokine signals. They found that Notch signaling directly bound to the Ifng locus to activate Ifng transcription independently of T-bet. ${ }^{9}$

Emerging data from recent studies indicate that DLL4 activation of Notch1 signaling is also important for proliferation of antigen-activated $\mathrm{CD} 8^{+} \mathrm{T}$ cells. ${ }^{19}$ These findings are in agreement with previous observation showing that Notch signaling is crucial for activating T-bet to promote the differentiation of $\mathrm{CD}^{+} \mathrm{T}$ cells into effector cells. ${ }^{54,55}$ Notch $1 / 2$ deficiency also reduced effector cell differentiation through impairing AKT and mTOR activation. ${ }^{9,54}$ Notch 1/2 
deficiency led to increased expression of transcription factors (Bc16, Foxo3, Foxo1, Tcf7, Id3), promoting memory precursor cell generation. ${ }^{55}$ It appears that Notch signaling has a broad impact on $\mathrm{CD}^{+} \mathrm{T}$ cell responses.

C.2. High binding affinity between DLL4 and Notch1/4. Better understanding of the molecular structure of DLL4 will be important for understanding why DLL4 has greater capacity than other Notch ligands to activate Notch signaling in $\mathrm{T}$ cells in the context of instructing their Th1 and Th17 differentiation. The human DLL4 gene was located on 15q21.1, and the mouse Dll4 gene was mapped to chromosome 2E3, a region that shows conservation of synteny with human chromosome $15 \mathrm{q} .{ }^{25}$ The open reading frame (ORF) of human $D L L 4$ is $\sim 86 \%$ identical at the nucleotide level and $87 \%$ identical at the amino acid level to murine Dll4. ${ }^{25}$ Like Notch receptors and other Notch ligands, DLL4 is composed of a module of N-terminal domain of Notch ligand (MNNL), followed by NSL domain, EGF-like repeats, transmembrane domain and intracellular domain (Figure 2). ${ }^{15,17,56}$ Unlike Jagged family Notch ligands, DLL4 has different numbers of EGF-like repeats and lacks an extracellular cysteine-rich domain and insertions that interrupt some EGF-like repeats. These structural differences categorize a Notch ligand as a Delta or Serrate family member. ${ }^{15,17,56}$ Besides, DLL4 is $\sim 50 \%$ identical to DLL1 at the amino acid level. ${ }^{57}$ These structure differences and the dramatic difference of amino acid sequence among Notch ligands may explain their functional differences.

DLL4 activates downstream Notch signaling mainly through Notch1 and Notch4 receptors. ${ }^{25,58}$ Coexpression of DLL4 and Notch1 or Notch4 induces the activation of target genes of Notch signaling in neural ectoderm in Xenopus embryos. In vitro binding affinity assay showed that 
DLL4 had an at least 10 fold higher binding affinity to Notch1 than DLL1. ${ }^{25}$ The molecular basis for DLL4 binding with Notch1 has been demonstrated with the analysis of crystal structure, further validating DLL4-Notch1 signaling pathway. ${ }^{58}$ Upregulation of Notch1 and Notch2 was seen in both $\mathrm{CD}^{+}$and $\mathrm{CD} 8^{+} \mathrm{T}$ cells after TCR activation, with clear increase of activated Notch1 NICD being detected. ${ }^{11,17,18,59}$ However, expression of Notch3 and Notch4 in activated T cells remains elusive. ${ }^{21}$ Our studies have shown that in vivo anti-DLL1 neutralizing antibody treatment did not affect IFN- $\gamma$ - and TNF- $\alpha$-producing $\mathrm{T}$ cells, indicating that DLL4-Notch signaling may play more important roles in vivo in $\mathrm{T}$ cell responses. ${ }^{14,19}$ Whether and how DLL4-Notch4 signaling regulates $\mathrm{T}$ cell immune responses remains to be explored. Report from other groups also found that blocking DLL4 in vivo had more dramatic effect in ameliorating GVHD and improving overall survival, further supporting this hypothesis. ${ }^{24}$

\section{MECHANISMS THAT REGULATE DLL4 ${ }^{+}$DC DIFFERENTIATION}

D.1. The role of Toll-like receptor (TLR) signaling in DC expression of DLL4. The capacity of different DC subsets to produce DLL4 under inflammatory conditions suggests that immature DCs may respond differentially to inflammatory stimuli in the context of upregulating DLL4. DCs become mature after encountering pathogens through activation of pattern recognition receptors including TLRs, Nod-like receptors (NLRs), C-type lectin receptors, mannose receptors and etc. ${ }^{60} \mathrm{CD} 1 \mathrm{c}^{+}$DCs express TLR4 and TLR7, whereas pDCs express TLR7 and TLR9 but lack TLR4. ${ }^{6,761-63}$ Data from our published studies indicate that while human immature CD1c ${ }^{+} \mathrm{DCs}$ and $\mathrm{pDCs}$ expressed low levels of DLL4, they rapidly upregulated the expression of DLL4 upon activation with TLR7/8 agonist R848 (Resiquimod) and / or TLR4 agonist LPS. Interestingly, monocyte-derived DCs (MoDCs) were unable to produce high levels 
of DLL4. MoDCs represent a subset of DCs of particular importance under inflammatory conditions, and have been widely used as vaccine adjuvants. ${ }^{6,64} \mathrm{We}$ found that both monocytes and their-derived MoDCs failed to produce high levels of DLL4 when they were stimulated with R848 and LPS. Real-time RT-PCR analysis further revealed that activated monocytes expressed 2 to 5-fold less $D L L 4$ transcripts compared to $\mathrm{pDCs}$ and $\mathrm{CD} 1 \mathrm{c}^{+} \mathrm{DCs}$. In contrast, MoDCs upregulated the expression of costimulatory molecules (e.g., CD40, CD80, CD83, and CD86), suggesting that the capacity of immature DCs to become DLL4 ${ }^{+}$DCs has been programmed during DC precursor development stages..$^{20}$ These observations indicate that the capacity of immature DCs to upregulate DLL4 has been established during the period of time when they are generated from their progenitor cells.

This hypothesis is further confirmed by our findings showing that human pDCs are composed of two distinct subsets in terms of their ability to produce DLL4 upon triggering TLR signaling. When being activated with high concentration of $\mathrm{R} 848, \mathrm{Lin}^{-} \mathrm{DR}^{+}$pan-DCs gave rise to approximately $90 \%$ of DLL4 ${ }^{+} \mathrm{CD} 1 \mathrm{c}^{+}$DCs and $35 \%$ of DLL4 $4^{+} \mathrm{pDCs}^{20}$ This allowed us to isolate pDCs into two subpopulations: DLL4 ${ }^{+}$pDCs and DLL4 ${ }^{-}$pDCs. When cocultured with allogeneic $\mathrm{CD}^{+} \mathrm{T}$ cells, we found that DLL4 ${ }^{+} \mathrm{pDCs}$ induced 2-fold more Th1 cells than DLL4- $\mathrm{pDCs}$. Interestingly, as compared to DLL4- $4^{-}$DCs, DLL4 ${ }^{+}$pDCs had higher levels of IL6, but were lower in $I L 1 B$ expression, two inflammatory cytokines important for Th17 cell differentiation. ${ }^{65}$ In addition, $\mathrm{DLL}^{+} \mathrm{pDCs}$ expressed 2-fold higher $I R F 8$, a transcription factor important for $\mathrm{pDC}$ development, compared to DLL4- pDCs. $^{66}$ This difference was unlikely the result of $\mathrm{pDC}$ activation, because both subsets showed similar levels of surface markers related to mature DCs (e.g., CD40, CD80, CD86 and CD83). ${ }^{66}$ 
Collectively, all these observations raise some important questions. For example, can DLL4 be used as a DC lineage marker for defining DC differentiation from hematopoietic progenitor cells (HPCs)? What is the intrinsic molecular mechanism that specifies the fate of DLL4 ${ }^{+}$DCs during hematopoietic progenitor cell differentiation and subsequently activate DLL4 transcription in activated immature DCs? Under what kind of environmental signals are HPCs driven to immature DLL4 ${ }^{+}$DCs?

D.2. Cytokine-mediated fate determination of immature DLL4 ${ }^{+}$DCs. $\quad$ To answer these questions, we have recently established a culture system to generate murine DLL4 ${ }^{+}$DCs from mouse bone marrow. ${ }^{19}$ Being cultured in the presence of FL, murine bone marrow (BM) cells differentiated into immature pDCs and cDCs, ${ }^{19}$ which were characterized by the surface


subsets were DLL4 negative, but rapidly upregulated the expression of DLL4 upon overnight incubation with LPS or R848. Concurrent stimulation with LPS and R848 induced much higher frequency of DLL4 ${ }^{+}$DCs. However, culture of BM cells in the presence GM-CSF failed to induce DLL4 ${ }^{+}$DCs despite stimulation with LPS and R848. ${ }^{19}$ These data suggest that the capacity of immature DCs to increase DLL4 upon inflammatory stimulation has been established during their differentiation from hematopoietic progenitor cells. These results are also in agreement with those aforementioned findings that upon GM-CSF culture human MoDCs were also unable to upregulate DLL4. Thus, FL promotes the generation of immature DLL4 ${ }^{+}$DCs from HPCs, whereas GM-CSF diverts the differentiation of HPCs into DLL4- DC subsets. 
Recent studies have demonstrated that inflammatory environment has significant impact on the generation of immature DLL4 ${ }^{+}$DCs and their differentiation into distinct DC subsets. ${ }^{12,68}$ Preparative conditioning for allogeneic HSCT may lead to increased release of LPS and other danger signals. ${ }^{69,70}$ The time kinetic analysis revealed that host-type DLL4 ${ }^{+}$DCs markedly increased 3 days after transplantation and declined by day 7. This is in agreement with previous observations that host DCs may finally diminish during the GVHD process. ${ }^{45,71-73}$ In mice undergoing HSCT, DCs can be de novo generated from infused donor BM cells. Interestingly, despite their exposure to inflammatory stimuli, newly generated donor origin DCs did not express DLL4 seven days after transplantation. It has been shown that seven days after HSCT in mice, alloreactive $\mathrm{T}$ cells differentiated into effector $\mathrm{T}$ cells that are able to produce inflammatory cytokines, including IFN- $\gamma$, TNF- $\alpha$ and GM-CSF. ${ }^{70}$ It is speculated that upon simulation with GM-CSF in vivo, donor HPCs may reduce their capacity to differentiate into DLL4 ${ }^{+}$DCs. We therefore propose that an impaired reconstitution of donor-type DLL4 ${ }^{+}$DCs in hosts undergoing allogeneic HSCT might reflect the presence of virulent GVH reactions that produce high levels of inflammatory cytokines such as TNF- $\alpha$ and GM-CSF.

\section{E. REGULATION OF DLL4 BY NFKB and STAT3 IN IMMATURE DCS}

Studies of mechanisms that induce DLL4 in DCs will be important for better defining the ontogeny of DLL4 ${ }^{+}$DCs and targeting these cells for immunotherapy. Our recently studies have demonstrated that Pam3 (TLR1/2 stimulus), Poly I:C (TLR3 stimulus), LPS and R848 induced high levels of DLL4 expression on the surface of $50 \%$ to $80 \%$ of CD1 $\mathrm{c}^{+} \mathrm{DCs}$, whereas IFN- $\alpha$ (pro-inflammatory cytokine) and CD40L (signal from activated $\mathrm{T}$ cells) did not. ${ }^{20} \mathrm{CpG}$ oligodeoxynucleotides (TLR9 agonists) did not increase DLL4 in CD1c ${ }^{+}$DCs,${ }^{20}$ consistent with 
the absence of TLR9. ${ }^{74,75} \mathrm{pDCs}$ increased DLL4 expression when activated by R848 $(16.0 \% \pm$ $2.7 \%)$ and to a lesser extent by $\mathrm{CpG}$ oligodeoxynucleotides $(8.6 \% \pm 0.8 \%) .{ }^{20}$ These results demonstrate that activation of TLR signaling induces high levels of DLL4 in CD1c ${ }^{+}$DCs and pDCs, with $\mathrm{R} 848$ being the most potent stimulus.

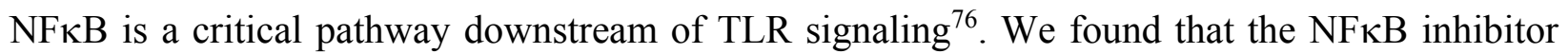
PDTC completely blocked DLL4 induction in both DC subsets, suggesting the important role of $\mathrm{NF} \kappa \mathrm{B}$ in inducing DC expression of DLL4. ${ }^{20}$ However, stimulation of monocytes with R848 +

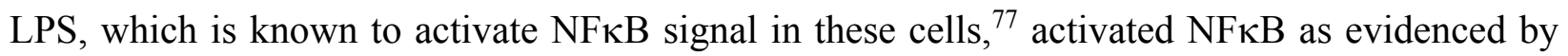
increased expression of phosphorylated P65 (p-P65), but induced low levels of DLL4 on the surface of monocytes. ${ }^{20}$ Furthermore, MoDCs derived from cultures in GM-CSF and IL-4 had elevated p-P65 following stimulation by R848 + LPS and but were DLL4 negative. ${ }^{20}$ These data suggest that activation of NFאB is important but not sufficient for inducing DLL4 in human DCs.

Many studies have demonstrated that STAT3 plays multiple roles in both innate and adaptive immunity. ${ }^{78-81}$ For example, STAT3 is essential for production of indoleamine-2,3-dioxygenase (IDO) by murine antigen-presenting cells,${ }^{80}$ thereby repressing $\mathrm{T}$ cell response. Conversely, data from clinical studies indicate that about half of these patients treated with a STAT3 inhibitor experienced pathogen-mediated diarrhea ${ }^{81}$, suggesting that STAT3 is required for protective mucosal immunity. In mice, loss of STAT3 results in deficiency of common DC precursors and their DC progenies, but has no effect on monocyte/macrophage differentiation of hematopoietic precursor cells. ${ }^{82}$ We found that activation of TLR7/8 signaling increased STAT3 and p-STAT3 in human PB CD1 $\mathrm{c}^{+}$DCs and pDCs. ${ }^{20}$ Inhibiting STAT3 led to dramatic decrease in expression 
of DLL4 transcripts and proteins in circulating DCs. Promoter reporter assay revealed that STAT3 activated DLL4 transcription. In contrast, monocytes or MoDCs expressed low levels of STAT3 mRNA and active p-STAT3 protein in response to R848 and LPS. ${ }^{20}$ This may explain the inability of monocytes and MoDCs to produce high level of DLL4. Thus, DC expression of DLL4 may have been developmentally programmed through a mechanism of permitting or repressing STAT3 transcription.

It has been shown that the interaction between $\mathrm{NF \kappa B}$ and STAT3 plays an important role in regulating inflammatory immune cells. ${ }^{78}$ This appears to be true in regulating the expression of DLL4 in human DCs. ${ }^{20}$ Freshly isolated CD1c $\mathrm{c}^{+}$DCs and pDCs expressed higher levels of STAT3 than MoDCs. Activation of TLR4/7 led to increased amount of both STAT3 and phosphorylated STAT3 (p-STAT3) in CD1 $\mathrm{c}^{+}$DCs and pDCs, but not in MoDCs. The increased level of STAT3 and p-STAT3 in these CD1 $\mathrm{c}^{+}$DCs and pDCs was accompanied by induction of high levels of DLL4 protein. Notably, inhibiting either STAT3 or NFאB caused a decrease in DLL4 expression in circulating CD1c $\mathrm{c}^{+} \mathrm{DCs}$ and $\mathrm{pDCs} .{ }^{20}$ These finding argue the notion that both $\mathrm{NF \kappa B}$ and STAT3 are required for the induction of DLL4 $4^{+}$DCs. It is likely that NFKB and STAT3 cooperate to activate DLL4 transcription in human DCs.

\section{F. DLL4 AND DLL4 ${ }^{+}$DCS IN T CELL IMMUNITY}

Autoimmunity. Several lines of evidence indicate that DLL4 and DLL4 ${ }^{+}$DCs may play important roles in regulating $\mathrm{T}$ cell immunity. DLL4 has important roles in the pathogenesis of autoimmune diseases. In a mouse model of respiratory syncytial virus (RSV)-exacerbated allergic airway disease, neutralizing DLL4 led to increased production of IL-4, enhanced Th2 
immunity the subsequent development of airway hyper-responsiveness. ${ }^{83}$ In contrast, in mice with multiple sclerosis (MS) induced by TMEV ${ }^{84}$ treatment with anti-DLL4 Ab resulted in significantly decreased expression of cytokines transcripts derived from Th1 and Th17 cells, reduced number of infiltrating mononuclear inflammatory cells in the spinal cords and suppression of the disease development ${ }^{84}$ A recent study further revealed that DLL4 influenced inflammatory T-cell response via modulating chemokine receptor expression in activated $\mathrm{T}$ cells during experimental autoimmune encephalomyelitis, thereby regulating migration of effector $\mathrm{T}$ cells. ${ }^{29}$ These observations indicate that DLL4 dual functions in T cell autoimmunity. It has a regulatory role in initiation on Th2 cytokine production and allergic responses. ${ }^{83}$ Furthermore, DLL4 activation of Notch signaling promotes Th1- and Th17 cell-mediated demyelinating disease. However, whether these effects result from DLL4 expressed by DCs or non-DCs are not defined in these studies.

It will be intriguing to determine whether DLL4 and DLL4 ${ }^{+}$DCs may be associated with autoimmune diseases in humans. Given the importance of $\mathrm{DLL4}^{+}$DCs in regulating inflammatory T cell response in HSCT patients and a variety of inflammatory disorders in mice, it is envisioned that DLL4 ${ }^{+}$DCs may have significant implications in the pathogenesis of autoimmune diseases in humans, such as lupus.

Tumor immunity. T cell immune responses play a central role in cancer immune surveillance, and the efficient induction of effector $\mathrm{T}$ cells against tumor antigens is required for successful immunotherapy for cancer patients. ${ }^{85,86}$ Data from a recent study has demonstrated that DLL4 on DCs is essential for an effective anti-tumor response. ${ }^{22}$ They observed that Notch signals 
enhanced $\mathrm{CD}^{+} \mathrm{T}$ cell priming, which could critically alter the course of immune response. In a model in which eradication of a tumor is dependent on cross-presentation of tumor $\mathrm{Ag}$ to $\mathrm{CD}^{+}{ }^{+} \mathrm{T}$ cells by host $\mathrm{MCH}-\mathrm{II}^{+}$APCs, they found that adoptively transferred Marilyn T cells responded to antigen derived from the tumor cells and constrained tumor growth in wild-type mice. ${ }^{53} \mathrm{In}$ contrast, the antitumor effect of Marilyn T cells was impaired in conditional DC-specific DLL4 deficient mice (DLL4 ${ }^{-/}$mice).$^{53}$ The inability of T cells to control tumor growth in the absence of DLL4 $^{+}$DCs were associated with less efficient stimulation of $\mathrm{CD}^{+} \mathrm{T}$ cell activation, metabolism, proliferation and cytokine secretion..$^{53}$ Other studies by Sugimoto et al. have demonstrated the importance of Notch signaling in mediating tumor immunity. ${ }^{87}$ Mice with Notch2-deficient-CD8 ${ }^{+} \mathrm{T}$ cells die earlier than control mice after inoculation with OVAexpressing EG7 thymoma cells. ${ }^{87}$ Furthermore, administration of anti-Notch2 agonist $\mathrm{Ab}$ augments tumor immunity. ${ }^{87}$ These findings are interesting as they imply that enhancing the presence of functional DLL4 ${ }^{+}$DCs in the lymphoid tissues and tumor sites might augment antitumor immunity.

Alloimmunity. GVHD is caused by donor T cells that recognize and react to alloantigens of the recipient. ${ }^{70}$ Upon APC activation, donor $\mathrm{T}$ cells become alloreactive effector $\mathrm{T}$ cells producing high levels of inflammatory cytokines (e.g., TNF- $\alpha$, IFN- $\gamma$, IL-4 and IL-17) and cytotoxic molecules, leading to host tissue injury and the subsequent life-threatening complication..$^{47,69,88-93}$ However, blockade of individual effector molecules has limited efficacy in controlling GVHD. ${ }^{69,93}$ We provide evidence for a function of DLL4 and DLL4 ${ }^{+}$DCs in eliciting allogeneic T-cell responses early during GVHD. ${ }^{14,19,20}$ As compared to DLL4- DCs, DLL4 ${ }^{+}$DCs had greater ability to stimulate the generation of alloreactive effector T cells producing IFN- $\gamma$ 
and IL-17. Blockade of DLL4 could abrogate this effect of DLL4 ${ }^{+}$DCs. Furthermore, in vivo administration of anti-DLL4 $\mathrm{Ab}$ caused a marked reduction of alloreactive effector $\mathrm{T}$ cells in GVHD target organs, leading to reduction of GVHD and significantly improved survival of mice after allogeneic HSCT. ${ }^{14}$ Our findings indicate that DLL4 ${ }^{+}$DCs and DLL4 are important for the generation of alloreactive effector $\mathrm{T}$ cells capable of mediating host tissue injury and could be beneficial targets for improving the efficacy of allogeneic HSCT. Elegant studies by Tran and colleagues have also demonstrated that inhibiting DLL4 and DLL1 blocked GVHD while preserving substantial anticancer activity in mice undergoing allogeneic HSCT. ${ }^{24}$ These findings establish that DLL4 and DLL4 ${ }^{+}$DCs are important for induction of GVHD.

Tissue resident APCs play critical roles in inducing organ-specific GVHD. ${ }^{44-47,94}$ Depletion of APCs in the spleen and liver reduced the GVHD in the liver but had no effect on the development of GVHD in the skin. ${ }^{45}$ Inhibiting Langerhan's cells in the skin resulted in inhibition of cutaneous GVHD. ${ }^{44}$ In our experimental studies, we found that DLL4 ${ }^{+}$DCs predominantly localized in the intestine of mice three days after allogeneic HSCT. In vivo administration of anti-DLL4 Ab dramatically reduced GVH reactions in the gastrointestinal (GI) tract, leading to significantly reduced GVHD in mice after allogeneic HSCT. ${ }^{14}$ This is in agreement with our recent observations that donor $\mathrm{T}$ cells lacking Notch signaling have drastically reduced ability to mediate intestinal GVHD. ${ }^{23}$ We proposed that DLL4 and intestinal DLL4 $^{+}$DCs could play important roles in mediating GVH reaction in these tissues. However, many studies have shown that DLL4 was expressed in inflammatory macrophages and endothelial cells in other models. ${ }^{13,17,29,95,96}$ Furthermore, GVHD still occurred after profound depletion of CD11 $\mathrm{c}^{+}$DCs in mice after allogeneic HSCT. ${ }^{71}$ More importantly, a recent study 
reports that non-hematopoietic APCs in the GI tract were important for production of GVHD in the local tissue. ${ }^{94}$ Thus, the impact of DLL4 blockade on T-cell responses in vivo should consider the potential contribution of DLL4-expressing non-hematopoietic APCs (such as endothelial cells and epithelial cells, etc.). Nevertheless, it will be intriguing to determine whether DLL4 ${ }^{+}$DCs may be sufficient to cause GVHD, and if non-hematopoietic APCs expressing DLL4 might have similar effect to DLL4 ${ }^{+}$DCs on promoting alloreactive effector Tcell responses.

Most recently, we have demonstrated that DLL4 ${ }^{+}$DCs can be used to program donor T cells to reduce their GVHD toxicity while retaining antitumor activity. ${ }^{19}$ Using an in vitro culture system, we activated donor $\mathrm{T}$ cells with allogeneic DLL4 ${ }^{+}$DCs and transferred them into allogeneic recipient mice. Mice receiving allogeneic DLL4 ${ }^{+}$DC-induced $\mathrm{CD}^{+} \mathrm{T}$ cells developed only minimal GVHD and complete survival. Importantly, these DLL4 ${ }^{+}$DC-induced alloreactive effector $\mathrm{T}$ cells had acquired the capability of killing leukemic cells. ${ }^{19}$ This may potentially improve the anti-leukemic response early after HSCT, and overcome some barriers to the GVL response such as high disease burden and pharmacologic immunosuppression. Given the fact that DC activation of naïve $T$ cells allows priming them with antigens, we propose that DLL4 ${ }^{+}$DCs loaded with leukemia-associated antigens may facilitate the selection and expansion of leukemic cell-reactive $\mathrm{T}$ cells that mediate anti-leukemia activity. Notably, we have recently identified human $\mathrm{DLL}^{+} \mathrm{DCs}$ that possess great ability to promote allogeneic $\mathrm{CD}^{+}$naïve $\mathrm{T}$ cells to become effector cells producing high levels of IFN- $\gamma \cdot{ }^{20}$ If sufficient numbers of human DLL4 ${ }^{+}$ DCs can be produced, a novel strategy can be potentially devised for human patients. 


\section{G. FUTURE PERSPECTIVE}

The recent years have seen major advances in our understanding of the impact of distinct DC subsets on inducing specialized $\mathrm{T}$ cell subsets both in mice and humans. ${ }^{14,19,20,22,43,48,54,63,97}$ $\mathrm{CD} 1 \mathrm{c}^{+} \mathrm{DCs}$ and $\mathrm{CD} 141^{+}$DCs have differential roles in the generation of $\mathrm{CD} 8^{+} \mathrm{T}$ cells with unique functional properties and tissue localization. ${ }^{43}$ Although both DC subsets from blood can induce efficient CTL immune responses, $\mathrm{CD} 1 \mathrm{c}^{+} \mathrm{DCs}$ from human lung have significant higher capacity than lung $\mathrm{CD} 141^{+}$DCs to induce the differentiation and expansion of local $\mathrm{CD} 103^{+} \mathrm{CD} 8^{+}$effector $\mathrm{T}$ cells. In addition, while $\mathrm{CD} 1 \mathrm{c}^{+} \mathrm{DCs}$ from lung and blood have been shown to promote Th17 generation, $\mathrm{CD} 141^{+} \mathrm{DCs}$ are more potent than $\mathrm{CD} 1 \mathrm{c}^{+} \mathrm{DCs}$ to induce IL4- and IL-13-producing Th2 differentiation after LAIV challenge. ${ }^{98,99}$ Moreover, human skin dermis $\mathrm{CD} 141^{+} \mathrm{DCs}$ are able to induce regulatory $\mathrm{CD} 4^{+} \mathrm{T}$ cells, which inhibit inflammation during allogneneic skin transplantation. ${ }^{100}$ It remains to be determined if the expression of DLL4 on the surface of $\mathrm{CD} 1 \mathrm{c}^{+} \mathrm{DCs}$ and $\mathrm{CD} 141^{+}$DCs may be important for them to regulate and maintain effector $\mathrm{T}$ cells in these local tissues where these DCs reside. Given that DLL4 expression is induced on the surface of DCs upon inflammatory stimulation, future studies will investigate whether DLL4 ${ }^{+}$DCs may be responsible for the induction of different subsets of specialized effector $\mathrm{T}$ cells in local tissues and whether DLL4 ${ }^{+} \mathrm{DCs}$ are associated with the development and progression of organ-specific inflammatory disorders, such as autoimmune disease and GVHD. Better defining the tissue distribution of DLL4 ${ }^{+}$DCs and their function in local tissues will be important to answer these questions.

Available data indicate that whether the immature DC can produce DLL4 upon inflammatory stimulation may have been established during differentiation of HPCs into immature DCs. ${ }^{20}$ DCs 
develop along a defined differentiation pathway from HSCs/HPCs, common myeloid progenitors (CMP), myeloid and DC progenitors (MDP), DC precursor cells and immature DCs. ${ }^{33,67,68}$ In response to inflammatory stimuli, immature DCs undergo maturation by increasing the expression of antigen-presenting molecules, costimulatory molecules and some cytokines. ${ }^{1,6}$ However, at which differentiation stages, including CMP, MDP, DC precursors and immature DCs, the fate of HSCs/HPCs to become DLL4 ${ }^{+}$DC precursor cells is established and why monocytes cannot become DLL4 ${ }^{+}$DCs, have yet to be determined. It is likely that DLL4 ${ }^{+}$DCs may be epigenetically programmed by environmental factors. Understanding of the epigenetic mechanism that regulates the generation of DLL4 ${ }^{+}$DCs may lead to new strategies to target DCs for improving the efficacy of cancer immunotherapies and controlling inflammatory disorders such as GVHD and autoimmune diseases.

Finally, since DLL4 ${ }^{+}$DCs play important roles in promoting the generation Th1 and Th17 cells, absence of DLL4 ${ }^{+}$DCs may lead to inadequate immune responses. After allogeneic HSCT, the reconstitution of DC network appeared to be attenuated both in patients and experimental mice. ${ }^{14,45,72,101-105}$ For example, in experimental mice, we found that while host DCs upregulated DLL4 early after transplantation, donor DCs reconstituted later in mice with GVHD did not express DLL4 despite the presence of potent inflammatory factors in vivo. In human patients, a substantial proportion of patients in clinic early after HSCT did not have or have very low level of circulating DLL4 ${ }^{+}$DCs. $^{20}$ Determining whether impaired generation of DLL4 ${ }^{+}$DCs may reflect the presence of severe GVHD and/or other co-morbidity and / or correlate with infection or relapse in patients may have significant implications in the improvement of immune 
reconstitution after allogeneic HSCT, chronic infection and cancer immunotherapies in a broad context. 


\section{Acknowledgments}

This work was supported by the American Cancer Society (YZ), Department of Defense (YZ) and NIH (CA172106,YZ; CA178202, RR) and NIH/NIAMS (R01AR061569, RC). 


\section{REFERENCES:}

1. $\quad$ Steinman, R.M. \& Banchereau, J. Taking dendritic cells into medicine. Nature 449, 419426 (2007).

2. Banchereau, J. \& Steinman, R.M. Dendritic cells and the control of immunity. Nature 392, 245-252 (1998).

3. Zamvil, S.S. \& Steinman, L. The T lymphocyte in experimental allergic encephalomyelitis. Annu Rev Immunol 8, 579-621 (1990).

4. Strioga, M.M., et al. Therapeutic dendritic cell-based cancer vaccines: the state of the art. Critical reviews in immunology 33, 489-547 (2013).

5. Palucka, K. \& Banchereau, J. Dendritic-cell-based therapeutic cancer vaccines. Immunity 39, 38-48 (2013).

6. Collin, M., McGovern, N. \& Haniffa, M. Human dendritic cell subsets. Immunology 140, 22-30 (2013).

7. Colonna, M., Trinchieri, G. \& Liu, Y.J. Plasmacytoid dendritic cells in immunity. Nature immunology 5, 1219-1226 (2004).

8. Benlahrech, A., et al. Human blood CD1c dendritic cells stimulate IL-12-independent IFN-gamma responses and have a strikingly low inflammatory profile. Journal of leukocyte biology (2015).

9. Bailis, W., et al. Notch simultaneously orchestrates multiple helper T cell programs independently of cytokine signals. Immunity 39, 148-159 (2013).

10. Skokos, D. \& Nussenzweig, M.C. CD8- DCs induce IL-12-independent Th1 differentiation through Delta 4 Notch-like ligand in response to bacterial LPS. J Exp Med 204, 1525-1531 (2007).

11. Amsen, D., Antov, A. \& Flavell, R.A. The different faces of Notch in T-helper-cell differentiation. Nat Rev Immunol 9, 116-124 (2009).

12. Amsen, D., et al. Instruction of distinct CD4 Thelper cell fates by different notch ligands on antigen-presenting cells. Cell 117, 515-526 (2004).

13. Mochizuki, K., He, S. \& Zhang, Y. Notch and inflammatory T-cell response: new developments and challenges. Immunotherapy 3, 1353-1366 (2011).

14. Mochizuki, K., et al. Delta-like Ligand 4 Identifies a Previously Uncharacterized Population of Inflammatory Dendritic Cells That Plays Important Roles in Eliciting Allogeneic T Cell Responses in Mice. J Immunol 190, 3772-3782 (2013).

15. Maillard, I., Fang, T. \& Pear, W.S. Regulation of lymphoid development, differentiation, and function by the Notch pathway. Annu Rev Immunol 23, 945-974 (2005).

16. Grabher, C., von Boehmer, H. \& Look, A.T. Notch 1 activation in the molecular pathogenesis of T-cell acute lymphoblastic leukaemia. Nat Rev Cancer 6, 347-359 (2006).

17. Radtke, F., Fasnacht, N. \& Macdonald, H.R. Notch Signaling in the Immune System. Immunity 32, 14-27 (2010).

18. Kopan, R. \& Ilagan, M.X. The canonical Notch signaling pathway: unfolding the activation mechanism. Cell 137, 216-233 (2009).

19. Mochizuki, K., et al. Programming of donor T cells using allogeneic delta-like ligand 4positive dendritic cells to reduce GVHD in mice. Blood (2016).

20. Meng, L., et al. The Notch Ligand DLL4 Defines a Capability of Human Dendritic Cells in Regulating Th1 and Th17 Differentiation. J Immunol 196, 1070-1080 (2016). 
21. Tran, I.T., et al. Blockade of individual Notch ligands and receptors controls graftversus-host disease. J Clin Invest 123, 1590-1604 (2013).

22. Laky, K., Evans, S., Perez-Diez, A. \& Fowlkes, B.J. Notch signaling regulates antigen sensitivity of naive CD4+ T cells by tuning co-stimulation. Immunity $\mathbf{4 2}, 80-94$ (2015).

23. Zhang, Y., et al. Notch signaling is a critical regulator of allogeneic CD4+ T-cell responses mediating graft-versus-host disease. Blood 117, 299-308 (2011).

24. Tran, I.T., et al. Blockade of individual Notch ligands and receptors controls graftversus-host disease. J Clin Invest (2013).

25. Shutter, J.R., et al. D114, a novel Notch ligand expressed in arterial endothelium. Genes Dev 14, 1313-1318 (2000).

26. Schaller, M.A., et al. Notch ligand Delta-like 4 regulates disease pathogenesis during respiratory viral infections by modulating Th2 cytokines. J Exp Med 204, 2925-2934 (2007).

27. Jang, S., Schaller, M., Berlin, A.A. \& Lukacs, N.W. Notch ligand delta-like 4 regulates development and pathogenesis of allergic airway responses by modulating IL-2 production and Th2 immunity. J Immunol 185, 5835-5844 (2010).

28. Fukushima, A., et al. Notch ligand Delta-like4 inhibits the development of murine experimental allergic conjunctivitis. Immunol Lett 121, 140-147 (2008).

29. Reynolds, N.D., Lukacs, N.W., Long, N. \& Karpus, W.J. Delta-like ligand 4 regulates central nervous system $\mathrm{T}$ cell accumulation during experimental autoimmune encephalomyelitis. J Immunol 187, 2803-2813 (2011).

30. Ito, T., et al. TLR9 regulates the mycobacteria-elicited pulmonary granulomatous immune response in mice through DC-derived Notch ligand delta-like 4. J Clin Invest 119, 33-46 (2009).

31. Schaller, M.A., et al. Delta-like 4 differentially regulates murine CD4 T cell expansion via BMI1. PLoS One 5, e12172 (2010).

32. Watowich, S.S. \& Liu, Y.J. Mechanisms regulating dendritic cell specification and development. Immunol Rev 238, 76-92 (2010).

33. Shortman, K. \& Naik, S.H. Steady-state and inflammatory dendritic-cell development. Nat Rev Immunol 7, 19-30 (2007).

34. Naik, S.H. Demystifying the development of dendritic cell subtypes, a little. Immunol Cell Biol 86, 439-452 (2008).

35. Reizis, B., Colonna, M., Trinchieri, G., Barrat, F. \& Gilliet, M. Plasmacytoid dendritic cells: one-trick ponies or workhorses of the immune system? Nat Rev Immunol 11, 558565 (2011).

36. Zuniga, E.I., McGavern, D.B., Pruneda-Paz, J.L., Teng, C. \& Oldstone, M.B. Bone marrow plasmacytoid dendritic cells can differentiate into myeloid dendritic cells upon virus infection. Nature immunology 5, 1227-1234 (2004).

37. Schlitzer, A., et al. Identification of CCR9- murine plasmacytoid DC precursors with plasticity to differentiate into conventional DCs. Blood 117, 6562-6570 (2011).

38. Kassner, N., et al. Cutting edge: Plasmacytoid dendritic cells induce IL-10 production in T cells via the Delta-like-4/Notch axis. J Immunol 184, 550-554 (2009).

39. Liou, L.Y., et al. In vivo conversion of BM plasmacytoid DC into CD11b+ conventional DC during virus infection. Eur J Immunol 38, 3388-3394 (2008).

40. Brown, P.G., et al. A 500-kiloton airburst over Chelyabinsk and an enhanced hazard from small impactors. Nature 503, 238-241 (2013). 
41. MacDonald, K.P., et al. Characterization of human blood dendritic cell subsets. Blood 100, 4512-4520 (2002).

42. Poulin, L.F., et al. Characterization of human DNGR-1+ BDCA3+ leukocytes as putative equivalents of mouse CD8alpha+ dendritic cells. J Exp Med 207, 1261-1271 (2010).

43. Yu, C.I., et al. Human CD1c+ dendritic cells drive the differentiation of CD103+ CD8+ mucosal effector T cells via the cytokine TGF-beta. Immunity 38, 818-830 (2013).

44. Merad, M., et al. Depletion of host Langerhans cells before transplantation of donor alloreactive T cells prevents skin graft-versus-host disease. Nat Med 10, 510-517 (2004).

45. Zhang, Y., et al. APCs in the liver and spleen recruit activated allogeneic CD8+ T cells to elicit hepatic graft-versus-host disease. J Immunol 169, 7111-7118 (2002).

46. Beilhack, A., et al. Prevention of acute graft-versus-host disease by blocking T-cell entry to secondary lymphoid organs. Blood 111, 2919-2928 (2008).

47. Shlomchik, W.D. Graft-versus-host disease. Nat Rev Immunol 7, 340-352 (2007).

48. Salmon, H., et al. Expansion and Activation of CD103(+) Dendritic Cell Progenitors at the Tumor Site Enhances Tumor Responses to Therapeutic PD-L1 and BRAF Inhibition. Immunity 44, 924-938 (2016).

49. Takeichi, N., et al. Ameliorating effects of anti-D114 mAb on Theiler's murine encephalomyelitis virus-induced demyelinating disease. Int Immunol 22, 729-738 (2010).

50. Mukherjee, S., Schaller, M.A., Neupane, R., Kunkel, S.L. \& Lukacs, N.W. Regulation of T cell activation by Notch ligand, DLL4, promotes IL-17 production and Rorc activation. J Immunol 182, 7381-7388 (2009).

51. Agarwal, P., et al. Gene regulation and chromatin remodeling by IL-12 and type I IFN in programming for CD8 $\mathrm{T}$ cell effector function and memory. J Immunol 183, 1695-1704 (2009).

52. Fang, T.C., et al. Notch directly regulates Gata3 expression during T helper 2 cell differentiation. Immunity 27, 100-110 (2007).

53. Newton, K., Sun, X. \& Dixit, V.M. Kinase RIP3 is dispensable for normal NF-kappa Bs, signaling by the B-cell and T-cell receptors, tumor necrosis factor receptor 1, and Tolllike receptors 2 and 4. Molecular and cellular biology 24, 1464-1469 (2004).

54. Mathieu, M., Duval, F., Daudelin, J.F. \& Labrecque, N. The Notch signaling pathway controls short-lived effector CD8+ T cell differentiation but is dispensable for memory generation. J Immunol 194, 5654-5662 (2015).

55. Backer, R.A., et al. A central role for Notch in effector CD8(+) T cell differentiation. Nature immunology 15, 1143-1151 (2014).

56. Sandy, A.R. \& Maillard, I. Notch signaling in the hematopoietic system. Expert opinion on biological therapy 9, 1383-1398 (2009).

57. Preusse, K., et al. Context-Dependent Functional Divergence of the Notch Ligands DLL1 and DLL4 In Vivo. PLoS Genet 11, e1005328 (2015).

58. Luca, V.C., et al. Structural biology. Structural basis for Notch1 engagement of Deltalike 4. Science 347, 847-853 (2015).

59. $\mathrm{Wu}, \mathrm{Y}$., et al. Therapeutic antibody targeting of individual Notch receptors. Nature 464, 1052-1057 (2010).

60. Takeuchi, O. \& Akira, S. Pattern recognition receptors and inflammation. Cell 140, 805820 (2010). 
61. Moseman, E.A., et al. Human plasmacytoid dendritic cells activated by CpG oligodeoxynucleotides induce the generation of CD4+CD25+ regulatory T cells. $J$ Immunol 173, 4433-4442 (2004).

62. Haniffa, M., et al. Human tissues contain CD141hi cross-presenting dendritic cells with functional homology to mouse CD103+ nonlymphoid dendritic cells. Immunity 37, 60-73 (2012).

63. Meixlsperger, S., et al. CD141+ dendritic cells produce prominent amounts of IFN-alpha after dsRNA recognition and can be targeted via DEC-205 in humanized mice. Blood 121, 5034-5044 (2013).

64. Segura, E. \& Amigorena, S. Inflammatory dendritic cells in mice and humans. Trends in immunology 34, 440-445 (2013).

65. Acosta-Rodriguez, E.V., Napolitani, G., Lanzavecchia, A. \& Sallusto, F. Interleukins 1 beta and 6 but not transforming growth factor-beta are essential for the differentiation of interleukin 17-producing human T helper cells. Nature immunology 8, 942-949 (2007).

66. Hambleton, S., et al. IRF8 mutations and human dendritic-cell immunodeficiency. The New England journal of medicine 365, 127-138 (2011).

67. Gilliet, M., et al. The development of murine plasmacytoid dendritic cell precursors is differentially regulated by FLT3-ligand and granulocyte/macrophage colony-stimulating factor. J Exp Med 195, 953-958 (2002).

68. Mildner, A. \& Jung, S. Development and function of dendritic cell subsets. Immunity 40, 642-656 (2014).

69. Ferrara, J.L., Levine, J.E., Reddy, P. \& Holler, E. Graft-versus-host disease. Lancet 373, 1550-1561 (2009).

70. Blazar, B.R., Murphy, W.J. \& Abedi, M. Advances in graft-versus-host disease biology and therapy. Nat Rev Immunol 12, 443-458 (2012).

71. Li, H., et al. Profound depletion of host conventional dendritic cells, plasmacytoid dendritic cells, and B cells does not prevent graft-versus-host disease induction. $J$ Immunol 188, 3804-3811 (2012).

72. Zhang, Y., Louboutin, J.P., Zhu, J., Rivera, A.J. \& Emerson, S.G. Preterminal host dendritic cells in irradiated mice prime CD8+ T cell-mediated acute graft-versus-host disease. J Clin Invest 109, 1335-1344 (2002).

73. Markey, K.A., MacDonald, K.P. \& Hill, G.R. Recipient plasmacytoid DCs are not required to prime allogeneic T-cell responses after BMT. Blood 113, 6038-6039 (2009).

74. Kadowaki, N., et al. Subsets of human dendritic cell precursors express different toll-like receptors and respond to different microbial antigens. $J$ Exp Med 194, 863-869 (2001).

75. Schreibelt, G., et al. Toll-like receptor expression and function in human dendritic cell subsets: implications for dendritic cell-based anti-cancer immunotherapy. Cancer immunology, immunotherapy : CII 59, 1573-1582 (2010).

76. Kawai, T. \& Akira, S. Signaling to NF-kappaB by Toll-like receptors. Trends in molecular medicine 13, 460-469 (2007).

77. Guha, M. \& Mackman, N. LPS induction of gene expression in human monocytes. Cellular signalling 13, 85-94 (2001).

78. Grivennikov, S.I. \& Karin, M. Dangerous liaisons: STAT3 and NF-kappaB collaboration and crosstalk in cancer. Cytokine \& growth factor reviews 21, 11-19 (2010).

79. Yu, H., Lee, H., Herrmann, A., Buettner, R. \& Jove, R. Revisiting STAT3 signalling in cancer: new and unexpected biological functions. Nat Rev Cancer 14, 736-746 (2014). 
80. Sun, Y., et al. Cutting edge: Negative regulation of dendritic cells through acetylation of the nonhistone protein STAT-3. J Immunol 182, 5899-5903 (2009).

81. Page, B.D., Ball, D.P. \& Gunning, P.T. Signal transducer and activator of transcription 3 inhibitors: a patent review. Expert opinion on therapeutic patents 21, 65-83 (2011).

82. Laouar, Y., Welte, T., Fu, X.Y. \& Flavell, R.A. STAT3 is required for Flt3L-dependent dendritic cell differentiation. Immunity 19, 903-912 (2003).

83. Okamoto, M., et al. Essential role of Notch signaling in effector memory CD8+ T cellmediated airway hyperresponsiveness and inflammation. J Exp Med 205, 1087-1097 (2008).

84. Takeichi, N., et al. Ameliorating effects of anti-D114 mAb on Theiler's murine encephalomyelitis virus-induced demyelinating disease. Int Immunol 22, 729-738.

85. June, C.H. Adoptive T cell therapy for cancer in the clinic. J Clin Invest 117, 1466-1476 (2007).

86. June, C.H. Principles of adoptive T cell cancer therapy. J Clin Invest 117, 1204-1212 (2007).

87. Sugimoto, K., et al. Notch2 signaling is required for potent antitumor immunity in vivo. $J$ Immunol 184, 4673-4678.

88. Martin, P.J., et al. Evaluation of mycophenolate mofetil for initial treatment of chronic graft-versus-host disease. Blood 113, 5074-5082 (2009).

89. Das, R., Chen, X., Komorowski, R., Hessner, M.J. \& Drobyski, W.R. Interleukin-23 secretion by donor antigen-presenting cells is critical for organ-specific pathology in graft-versus-host disease. Blood 113, 2352-2362 (2009).

90. Cutler, C. \& Antin, J.H. Novel drugs for the prevention and treatment of acute GVHD. Curr Pharm Des 14, 1962-1973 (2008).

91. Chao, N.J. Are there effective new strategies for the treatment of acute and chronic GvHD? Best Pract Res Clin Haematol 21, 93-98 (2008).

92. Deeg, H.J. How I treat refractory acute GVHD. Blood 109, 4119-4126 (2007).

93. Blazar, B.R. \& Murphy, W.J. Bone marrow transplantation and approaches to avoid graft-versus-host disease (GVHD). Philos Trans R Soc Lond B Biol Sci 360, 1747-1767 (2005).

94. Koyama, M., et al. Recipient nonhematopoietic antigen-presenting cells are sufficient to induce lethal acute graft-versus-host disease. Nat Med 18, 135-142 (2011).

95. Mailhos, C., et al. Delta4, an endothelial specific notch ligand expressed at sites of physiological and tumor angiogenesis. Differentiation 69, 135-144 (2001).

96. Gale, N.W., et al. Haploinsufficiency of delta-like 4 ligand results in embryonic lethality due to major defects in arterial and vascular development. Proceedings of the National Academy of Sciences of the United States of America 101, 15949-15954 (2004).

97. Nizzoli, G., et al. Human CD1c+ dendritic cells secrete high levels of IL-12 and potently prime cytotoxic T-cell responses. Blood 122, 932-942 (2013).

98. Schlitzer, A., et al. IRF4 transcription factor-dependent CD11b+ dendritic cells in human and mouse control mucosal IL-17 cytokine responses. Immunity 38, 970-983 (2013).

99. Yu, C.I., et al. Human CD141+ dendritic cells induce CD4+ T cells to produce type 2 cytokines. Journal of immunology 193, 4335-4343 (2014).

100. Chu, C.C., et al. Resident CD141 (BDCA3)+ dendritic cells in human skin produce IL-10 and induce regulatory $\mathrm{T}$ cells that suppress skin inflammation. The Journal of experimental medicine 209, 935-945 (2012). 
101. Bossard, C., et al. Plasmacytoid dendritic cells and Th17 immune response contribution in gastrointestinal acute graft-versus-host disease. Leukemia 26, 1471-1474 (2012).

102. Fearnley, D.B., Whyte, L.F., Carnoutsos, S.A., Cook, A.H. \& Hart, D.N. Monitoring human blood dendritic cell numbers in normal individuals and in stem cell transplantation. Blood 93, 728-736 (1999).

103. Reddy, V., et al. Low dendritic cell count after allogeneic hematopoietic stem cell transplantation predicts relapse, death, and acute graft-versus-host disease. Blood 103, 4330-4335 (2004).

104. Stenger, E.O., Turnquist, H.R., Mapara, M.Y. \& Thomson, A.W. Dendritic cells and regulation of graft-versus-host disease and graft-versus-leukemia activity. Blood 119, 5088-5103 (2012).

105. Waller, E.K., et al. Improved survival after transplantation of more donor plasmacytoid dendritic or naive T cells from unrelated-donor marrow grafts: results from BMTCTN 0201. Journal of clinical oncology : official journal of the American Society of Clinical Oncology 32, 2365-2372 (2014). 


\section{Figure legend}

Figure 1. DLL4 activation of Notch signaling in T cells. DLL4 derived from DCs activates Notch receptor expressed on the surface of T cells, and triggers Notch cleavage by the ADAM metalloproteases and $\gamma$-secretase complex, generating active intracellular domain of notch (NICD). This leads to release of NICD from cytoplasm into nucleus, after which NICD binds to the DNA-binding protein complex CSL/RBPj. Interaction of NICD with CSL leads to the release of co-repressor which are inhibitory interaction proteins with CSL when there is no NICD in the nucleus, followed by recruitment of transcriptional co-activator including MAML and others. NICD, CSL and MALM and other co-activator form a complex and induce the active transcription of downstream Notch targets (e.g., GATA3, T-bet, Rorc, ec.). Following TCR and costimulatory signaling induced T cell activation, DLL4 activation of Notch signaling is crucial for instructing the development of Th1, Th17 and cytotoxic T lymphocytes (CTL).

Figure 2: Biochemistry of Notch ligands and Notch receptors. There are five Notch ligands (Jagged 1, Jagged2, DLL1, DLL3 and DLL4) and four Notch receptors (Notch 1, 2, 3 and 4). Both Notch ligands and Notch receptors are transmembrane proteins, containing extracellular domain, transmembrane domain and intracellular domain. EGF-like repeats constitutes the majority of extracellular domain for both ligands and receptors, with each receptor and ligand having different number of repeats specified as indicated. Noteworthy, although DLL1 and DLL4 have almost same structure, they are only $\sim 60 \%$ identical in protein sequence. The situation for Notch1 and Notch2 is the same. The interaction region for DLL4 ligand and Notch1 include most of the extracellular domain of DLL4 and some of the EFG-like repeats of Notch1 extracellular domain as indicated in this figure in bright green shade. Each domain is indicated in the right corner. 
Graphical Abstract

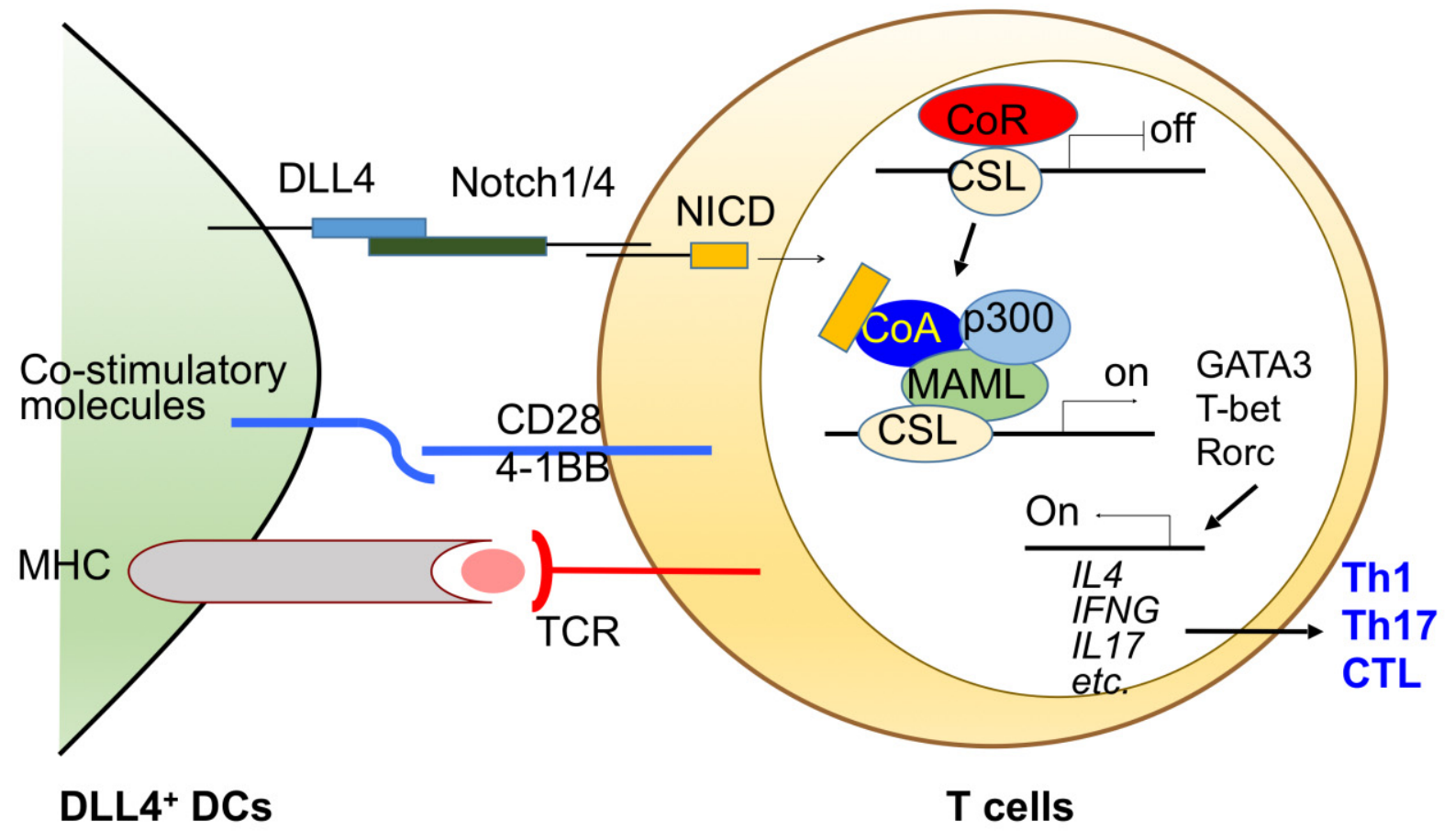






Fig.1 
